\title{
Enhancing Decisions in Prognostics and Health Management Framework
}

\author{
Omar Bougacha ${ }^{1}$, Christophe Varnier ${ }^{2}$ and Noureddine Zerhouni ${ }^{3}$ \\ 1,2,3 FEMTO-ST, AS2M, \\ Univ. Bourgogne Franche-Comté, ENSMM, \\ Besançon, 25000, France \\ omar.bougacha@femto-st.fr \\ christophe.varnier@femto-st.fr \\ noureddine.zerhouni@femto-st.fr
}

\begin{abstract}
Prognostics and health management have become increasingly important in recent years. Many research studies focus on a crucial phase consisting of predicting the remaining useful life of equipment or a component. However, this step is often carried out without taking into account the decisions that will be taken later. This article aims to propose a modification of the existing PHM framework to combine the prognostics and decision-making phases in a closed loop. In this paper, the presented framework is described and some elements for its implementation are proposed. A simplified example is developed to illustrate the presented methodology of post-prognostic decision enhancement.
\end{abstract}

\section{INTRODUCTION}

The growing need of the industry for the high reliability, availability and operation safety of its systems was the root of the maintenance evolution. During the last decades, maintenance policies evolved from corrective maintenance, in which interventions are only made after the failure occurrence, to preventive maintenance with predefined maintenance dates based on the component's reliability information like the mean time between failures. Later, the conditionbased maintenance (CBM) strategy appears thanks to the evolution of condition monitoring and health assessment technologies. With CBM, the degradation or the health of a component is monitored and intervention is planned when the level of the degradation exceeds a certain threshold. Recently, and with the emergence of the prediction techniques (Vachtsevanos \& Wang, 2001), the era of predictive maintenance began via prognostics and health management (PHM) (Byington, Roemer, \& Galie, 2002). In the predictive main-

\footnotetext{
Omar Bougacha et al. This is an open-access article distributed under the terms of the Creative Commons Attribution 3.0 United States License, which permits unrestricted use, distribution, and reproduction in any medium, provided the original author and source are credited.
}

tenance context, the maintenance decisions are about setting intervention dates based on the remaining time during which the system can still fulfill its purpose. Such a metric is called remaining useful life (RUL) and it is one important metric of the PHM framework. The PHM was based on the functionalities of CBM. Therefore, its framework was defined similarly to CBM. Lebold and Thurston, defined in (Lebold \& Thurston, 2001), the open system architecture for condition-based maintenance and prognostic systems. The defined framework is considered as fundamental for all PHM applications. And it is widely used in the literature.

One can find a lot of definitions for the PHM concept in literature, but no consensual definition has yet been proposed. Some authors like Uckun et al. in (Uckun, Goebel, \& Lucas, 2008), defined it as an engineering discipline that studies the failure mechanism and manages the systems life cycle. Skima in (Skima, 2016) added that its main objective is to minimize the operational and maintenance costs. Sun et al. in (Sun, Zeng, Kang, \& Pecht, 2010), considered PHM as a methodology to predict when and which component will fail and manage the system's reliability. Goebel et al. in (Goebel et al., 2017), presented PHM as the procedure of studying the conditions of an engineering system, whether its behavior is within predefined nominal boundaries and in case of a deviation, predicts where and when the system would fail. Based on this information, adequate decisions are taken to mitigate the effects of an undesirable event. Considering these definitions PHM can be defined as an engineering discipline that investigates the reliability of a system and manages its conditions through a set of tools, methods, and processes that performs health assessment, diagnostics, prognostics, and decision-making.

For many years, the studies of the PHM process were limited to some methods or tools that enhanced the health assessment, diagnostics, and prognostics functions. One can 
refer to some of the reviews of the works done in diagnostics (Gertler, 2013), (Wu, Vachtsevanos, Lewis, Roemer, \& Hess, 2006), (Tamilselvan \& Wang, 2013), health assessment (Kandukuri, Klausen, Karimi, \& Robbersmyr, 2016) and particularly prognostics (Schwabacher, 2005), (Si, Wang, Hu, \& Zhou, 2011) and (An, Kim, \& Choi, 2015). Later on, the health management term of the PHM was introduced by integrating the estimated remaining useful life (RUL) in the decision-making process. As described by Goebel et al. in (Goebel et al., 2017), prognostics is the science of making predictions but the estimation of the RUL is not performed as an end of PHM in itself, but for improving the decisionmaking process. Therefore, the notion of post-prognostic decision-making is an important phase in the PHM framework.

One can find several definitions of post-prognostics decisions, (Goebel, Iyer, \& Bonissone, 2006), (Balaban \& Alonso, 2012), (Herr, 2015), all these references stated that postprognostic decisions are the result of integrating the health indicators and/or the RUL in the decision-making process. The decision-making process is subject to an optimization function that best serves the intended application and may include the health state of the system. For a long period, postprognostic decision-making was only dealing with maintenance decision-making. Gouriveau et al. in (Gouriveau, Medjaher, \& Noureddine, 2016), stated that the application of PHM should not be limited to industrial maintenance and that PHM is considered to be more general and could be applied to different activities by diversifying the nature of the decisions to be taken. Nowadays, decisions in the PHM context are more involving operational aspects of the studied systems like the production scheduling (Ladj, Varnier, Tayeb, \& Zerhouni, 2017), the mission assignment (De Medeiros, Rodrigues, Santos, Shiguemori, \& Júnior, 2014), control decisions (Pereira, Kawakami, Galvao, \& Yoneyama, 2010), etc. Moreover, some works in literature combined the two aspects of decisions (maintenance and operational) leading to a new category of decision that we noted mixed decisions (Cheng, Zhou, \& Li, 2018), in which simultaneously the maintenance interventions and the operational controls are optimized.

By focusing on post-prognostic decision-making in the previous works, we came cross some major omissions:

- How do the RUL uncertainties influence the decision process?

- How do the works on post prognostic decisions integrate the RUL in the process?

- What is the decision horizon duration? How does this value influence the results? Based on what criteria the duration is determined?

- How frequently is the PHM process launched? Is it periodically launched or event-driven?
- Do the prognostics algorithms take future decisions into considerations?

- How the new evolution of the system's health state is integrated into the prognostics algorithm?

These oversights are more detailed in the next section. The main aim of this paper is to question these points. Moreover, the paper presents a methodology to enhance decisionmaking in the PHM framework. Some minor adjustments of the OSA-CBM framework are proposed in this aim.

This paper is organized as follows. In the first section, we propose an overview of the PHM processes while focusing on the main lacks of the post-prognostics decision process. In section 3, the definition of some terms, that are needed to guarantee the consistency of the proposed framework, is given. Section 4 contains the adaptation of the PHM framework to enhance decision-making. In section 5, an explanatory example is given to defining the different elements of the resolution. This example is just an illustration to validate the proposed methodology. Finally, a conclusion and some future works are presented in section 6.

\section{Classical PHM and Motivation}

As presented in the OSA-CBM (Lebold \& Thurston, 2001), PHM for a specific system embeds seven levels presented in Figure 1. This seven layers framework explains the PHM process from sensors data acquisition to decision-making and human-machine interface. Lately, these layers have been grouped into three phases by Gouriveau et al. in (Gouriveau et al., 2016). The observation phase starts with the sensors' data acquisition, the stored data is then processed through filtering, modifying and feature extraction. In the analysis phase, the system's conditions are monitored through the preprocessed features, its heath state is assessed, some fault diagnosis is performed if needed and the systems remaining useful life is estimated in the prognosis process. Finally, the action phase involves decision-making and different information visualization in the Human-Machine Interface. Therefore, we can model a global PHM process with a block diagram as shown in Figure 2. Moreover, the proposed presentation is used all along this work to guarantee the consistency of the paper. Figure 2 describes the process of PHM. The system can produce data coming from sensors or observations. The obtained data allows the monitoring of the health and the degradation of certain components that are in most cases the cause of the system's failure. Through the phase of observation, the sensors data will be acquired, stored and processed to obtain the features that describe the system health state through health indicator (HI) and the evolution of degradation of its components. The obtained features are feed to the analysis phase, in which the health conditions are monitored to detect the beginning of a degraded pattern. The health state of the system is assessed and the remaining 


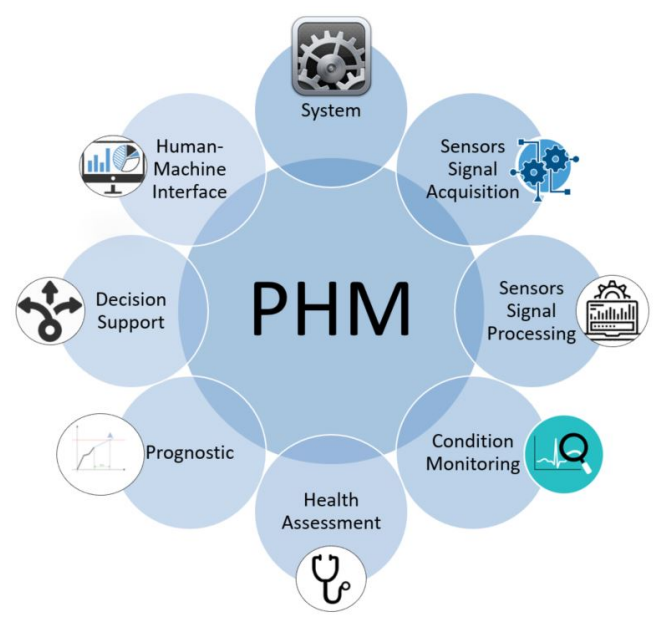

Figure 1. The PHM process as presented in the OSA-CBM

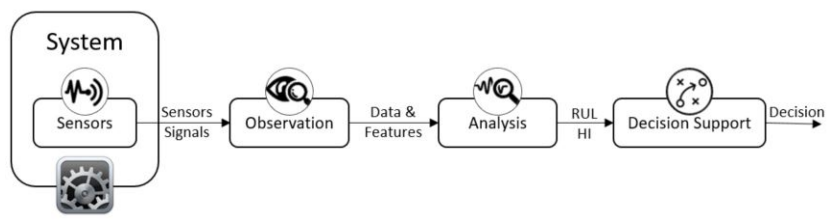

Figure 2. Block Diagram of classic PHM

useful life is predicted. Based on the RUL values, decisions are selected. Moreover, the made decisions are applied to the system.

The term management in PHM refers to the post-prognostic decision-making process (Goebel et al., 2017). The process consists of solving an optimization problem for production scheduling, maintenance planning, control parameters adjusting, mission assignment, etc. while integrating prognostics information. In the classic PHM context, authors generally try to optimize the maintenance schedule to minimize the cost and avoid failures. Yet several works in literature dealt with post-prognostics decisions and other decisions types than maintenance scheduling are taken. By considering the type of the proposed decisions, one can classify them into three categories:

- Maintenance Decisions: In which, the remaining useful life of components or system is used to define the suitable maintenance date for each component. These decision are wildly studied in several industrial domains: transport (Rodrigues et al., 2015), manufacturing (Yang, Djurdjanovic, \& Ni, 2008), (Van Horenbeek \& Pintelon, 2013), (Do, Voisin, Levrat, \& Iung, 2015), (Liu, Dong, Lv, \& Ye, 2017), aerospace (Balaban \& Alonso, 2012) (Goebel et al., 2006), Wind-Turbines (Lei \& Sandborn, 2016).

- Operational Decisions: One can also modify the operational parameters of the system to better manage its health state. The operational decisions can be divided into three subcategories:

- Production and Tasks assignment: It consists of scheduling production activities or defining a good match between the tasks to be done and the system degradation. Such decisions are mostly studied in manufacturing (Skima, Varnier, Dedu, Medjaher, \& Bourgeois, 2017), path planning for UAVs fleets (De Medeiros et al., 2014) or train rolling stocks.

- Control Decisions: Some control loops use health monitoring of the actuator in the adjustment of the controller parameters. Moreover, some works adapt the controller setpoints according to the actuator health state ((Pereira et al., 2010), (Langeron, Grall, \& Barros, 2015)).

- Logistics: The needs of the system in terms of raw materials and spare parts are determined by its health state. Thus, the provisions, the orders, and the logistic movements are made based on the monitored condition and the RUL of the system to reduce the costs of storage and the penalties on waiting in case of shortage in raw materials or spare parts. Logistic decisions take into consideration the health condition of the system, the lead time of ordering and the storage levels to optimize the cost (Cui, Shi, \& Wang, 2015), (Lin, Basten, Kranenburg, \& van Houtum, 2017).

- Mixed Decisions: In this category, one considers both sides of the system functional requirement i.e. the operational and the maintenance requirements. Therefore, some works focused on jointly optimizing production and maintenance like Fitouri et al. in (Fitouri, Fnaiech, Varnier, Fnaiech, \& Zerhouni, 2016). Other authors, like De Medeiros et al. (De Medeiros, Rodrigues, Kern, dos Santos, \& Shiguemori, 2015) tried to find a compromise between carrying out a mission and maintaining the system. More authors considered the ordering of spare parts while defining the maintenance dates to optimize the spare part storage cost (Wang, Hu, Wang, Kong, \& Zhang, 2015), or even integrate quality control and maintenance interventions (Cheng et al., 2018).

In the PHM context, decisions are made and applied to the system. Thus, these decisions change the evolution of the systems' heath state. For example, Frost et al. (Frost, Goebel, Frost, Trinh, \& Balas, 2013) used the health state of wind turbine blades in an adaptive controller to extend the operating time of the system. This can be observed through the modification in the available sensors data (noted S_Signals' in figure 3 ) and the new trends that appear in the preprocessed data and the associated features. The new trends in the features describe a new evolution of the system health states. This evolution may be different from the evolution of the features used to estimate the first remaining useful life (noted RUL). 
Thus, a new remaining useful life is estimated (noted RUL'). Therefore, to best fulfill the objective of the framework, the decision process must be adapted to include the current prognostic information. This feedback of the decision made on the system is represented by the red dashed line in figure 3 . Although this process is natural, it is not explicitly mentioned in most of the literature works. The previous works in literature, are limited to making decisions on a finite fixed decision horizon. In most of the cases, the considered decision horizon is long enough to incorporate more than one maintenance action, this is the case in the work of Camci, (Camci, 2009). After the first maintenance intervention, the authors, usually, use reliability rules on the remaining decision horizon to model the system behavior. These works implicitly assume that the used prognostics methods are accurate and precise. They also assume that a maintenance action will be performed at the exact scheduled moment with the suitable quality. Several works included future decisions in the prognostics algorithms, for example in (Sierra, Orchard, Goebel, \& Kulkarni, 2019) authors used future power consumption profiles in the prognostics algorithm to predict the end of discharge of a Lithium-polymer battery in a rotatory-wing UAV. Although prognostics algorithms take into consideration variable future conditions, most works on post-prognostic decisions (for example (Camci, 2009), (Lei \& Sandborn, 2016), and (Huynh, Grall, \& Bérenguer, 2017)) generally assume that these future conditions are constant during the decision horizon. This implies a highly controlled environment of the system which can only be achieved in a laboratory. More generally, only a single RUL value is estimated and used for the decision-making process.

Indeed, the prognostic module is highly dependent on the future usage conditions of the system and future decisions. Actually, in (Goebel et al., 2017), Goebel et al. presented various kinds of uncertainties that can influence the RUL value. Two particular uncertainties are related to the future loads of the system and its environmental conditions. In the rest of the paper, we define the operating conditions as the fusion of future loads and environmental conditions. One can also find some works in the literature about integrating future loads in the prognostic algorithm. For instance, Daigle and Goebel (Daigle \& Goebel, 2010) and Zhang et al. (Zhang, Tang, Decastro, Roemer, \& Goebel, 2014) used a model-based prognostic method and they considered the future load data in the procedure of estimating the RUL. Other works combined different modeling tools with the prognostics algorithm to integrate the future decision, like Welz et al. in (Welz, Coble, Upadhyaya, \& Hines, 2017) in which the authors integrated maintenance information into the prognostics module by combining the Weibull reliability model and the general path methods, Vileiniskis and Remenyte Prescott (Vileiniskis \& Remenyte-Prescott, 2017), on the other hand, used Petri-net to model the system, its

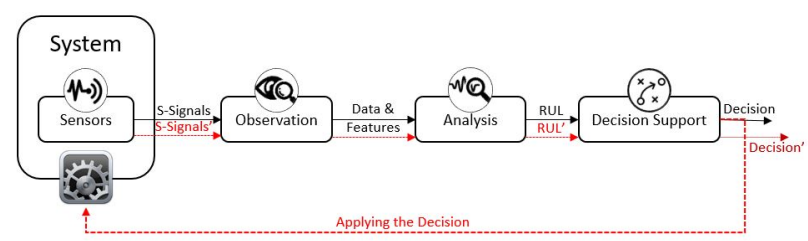

Figure 3. Dynamics of the PHM process

degradation and its future load, and maintenance actions. They ran a Monte Carlo simulation to obtain the statistics of components performance over a selected horizon. However, several works on prognostics algorithms do not consider the effects of future loads evolution on the remaining useful life. One can cite as an example the works of Dong et al. (Dong, Jin, Lou, \& Wang, 2014), and Mosallam et al. (Mosallam, Medjaher, \& Zerhouni, 2016).

On the other hand, the influence of the RUL on making decisions has been introduced in the literature through different methods of integrating the prognostic information in the decision process. Goebel et al. in (Goebel et al., 2017), explained that the possible actions to take in the decision-making process depend on the prognostic horizon, which was defined by the RUL of the system. Therefore, the RUL was used as a classification criterion to limit the decision search space. The works in literature integrated the RUL with different levels in the decision-making process. Some works used it as a constraint to define the system maintenance dates like in $(\mathrm{Cai}, \mathrm{Li}$, $\&$ Chen, 2016) in which the authors optimized the maintenance cost of an airplane by finding the suitable maintenance date based on the aero-engine RUL. While others used it as a predefined decision variable that limits the production profile and the horizon of production of the machine, like what was proposed by Herr et al. in (Herr, Nicod, \& Varnier, 2014). They try to vary the different production profiles of the machines to maximize the production horizon while satisfying a constant demand. Few works used the RUL in the optimization objective function, like Zhang et al. in (Zhang et al., 2014) when they planned the path for an autonomous vehicle in a way to save its battery energy consumption and to reduce its degradation. Fewer fully integrated the RUL as a variable to define the control parameters of an actuator as presented in (Langeron, Grall, \& Barros, 2013) by redefining the LQR control parameters with the evolution of the RUL. This proves that decisions in the context of PHM are highly dependent on the estimated RUL value. One can outline the integration of RUL in the decision-making process into four levels:

- As a classification criterion to select an option from a predefined range of decision, or to simply schedule maintenance intervention if the RUL exceeds a threshold. Khoury et al. (Khoury, Deloux, Grall, \& Bérenguer, 2013) used the estimated RUL to select the suitable maintenance opportunity. 
- As a constraint by considering a threshold above which the system is not allowed to function. In this case, the system actions are assumed to have a known degradation rate. In the work of Herr et al. (Herr et al., 2017) each trip has its degradation rate on the system. The sum of the degradation rates for a train is constrained to be lower than a threshold so that the schedule is considered feasible.

- As a penalty in the objective function to add a certain level of sensibility to the degradation rate of the decisions (Zhang et al., 2014) or to reduce the lost remaining useful life due to early maintenance et al. (Liu et al., 2017).

- As a variable to define new settings of control. This is very commonly used in the works that considered adapting the predictive controller's parameters to the health state of the actuator such in Pereira et al. (Pereira et al., 2010).

To summarize, the prognostic and the decision-making modules are highly inter-dependent. The prognostic module influence the decision support system and its output with the RUL value. Besides, the selected decisions can change the systems state and affect on the degradation evolution, and thus modify the remaining useful life. Moreover, integrating the decision in the form of future loads in the prognostic procedure can improve the RUL precision and reduce the level of the uncertainties. These processes are often considered sequentially and studied separately. Some works have considered this point. This is the case of Zhang et al. (Zhang et al., 2014). However, the processes were used sequentially. Once a decision is selected it is applied to the system, then a new RUL is estimated to select the next decision. Although this approach highlights the importance of considering future decisions in the prognostic algorithm, it assumes that a sequence of local optimal decisions leads to a global optimal decision. This approach makes decisions on a single step ahead and does not allow to question the previous decision quality. This paper aims to emphasize the interactions between decision-making and prognostics by considering both processes simultaneously. To improve the RUL estimations and provide more coherent decisions, these processes are adjusted to construct decisions over multiple steps ahead simultaneously while estimating their outcomes on the evolution of the system's health state. The proposed framework allows to question the relevance of previously selected decisions before applying them on the system.

Another major challenge in the PHM context is the used data for the prognostics modeling and training (in the case of data-driven and hybrid prognostic methods). As it has been proven, degradation data are highly related to the operation conditions of the system. For example, in the data generated by PRONOSTIA (Nectoux et al., 2012) for rolling bearing, the speed of rotation has a major influence on the degradation profile. Therefore, using data from historic degradation can be a good starting point for the prognostic process. But if such a prognostic process is still in use under new operating conditions, it will lead to questionable outcomes. Therefore, there is a need to incrementally update the process. Several approaches could be used for these updates such as case-based reasoning, reinforcement learning, etc.

In post-prognostic decision-making literature, the decision is directly made over a long duration. Moreover, in most of the works, the future decisions and their influence on the systems health state are not considered in the decision-making process nor the prognostic module. For example, one can see the works of Van Horenbeek and Pintelon (Van Horenbeek \& Pintelon, 2013), Wang et al. (Wang et al., 2015), and de Medeiros et al. (De Medeiros et al., 2014). The parameters of the prognostic method are not updated with new data that are obtained under unknown operating conditions. To keep the framework consistent with any observed changes in the system operations, the parameters of the prognostic algorithm should be updated when unknown conditions occur. However, to our knowledge, this point was not discussed in most works and the question of how does the prognostic method react to system changes remains unanswered. Therefore, one can qualify the PHM framework in this case as an open-loop process. To answer these challenges, the purpose of this work is to provide a new adaptation to the PHM framework to upgrade it to a closed-loop process. The proposed framework allows the construction of decisions while integrating their effects on the system's health state evolution in the remaining useful life estimation process. The interactions between prognostics and decision-making are modeled by building decisions iteratively over the duration of the considered horizon. Also, the data used as a reference for the prognostic module are refined to include new variation in the system behavior to guarantee a consistent prognostic method towards systems' changes. The Focus of the adaptation of the framework is on the decision-making process to improve the integration and the quality of the prognostic information. Therefore, in the paper, we concentrated more on post-prognostic decisions, but some elements of thoughts are proposed for the prognostic part. Although the proposition aims to improve certain points of the PHM framework, as discussed above, it remains with certain limits based on the following major assumptions:

- The data from the system sensors can be processed and allows to obtain suitable health indicators.

- The system health state and degradation are supposed to be observable to a certain extent. Meaning, that from sensors acquired data, one can estimate with a measurable confidence the actual health state or degradation level of the system.

- The degradation of the system is mastered to some extent. In other words, one can model the degradation through physics-based models, stochastic models, datadriven models or hybrid models 
- The matter of adequate prognostic methods is not addressed in this paper, under the assumption that adequate methods exist for short and long term prognosis.

To better introduce the proposed framework, some essential definitions are presented in the next section.

\section{DEFINITIONS}

\subsection{Elementary Action}

In a PHM context while stating the decision problem, there is a need to define the possible actions that can be applied. These actions are called, in this context, Elementary Actions. One can define an elementary action $\alpha_{i}$ as a countable set of descriptors $d_{i, j}$ (attribute $a_{j}$, value $v_{i, j}$ ). Attributes can be for instance:

- The type of action: The elementary action can either be an operational decision as in automatic control, scheduled production order, assigned mission, supply chain management action, or maintenance of a component, subsystem or the whole system.

- The concerned system, or component: Here, one defines the part of the system concerned by the proposed action.

- The task: In the case of mission assignment, production scheduling, or supply chain management, the task to execute, or the job the schedule for production is defined here.

- The control parameters: In the case of automatic control, this field is used to define the values of the control parameters. For example in (Langeron et al., 2013), this attribute corresponds to the new values of the $\mathrm{Q}$ and $\mathrm{R}$ matrix of the used LQR controller.

- The new control set-points: Still in the case of automatic control, one can modify the set-points of the controllers and therefore the new reference values are defined here.

- Duration: The duration of the action needs to be defined or estimated if no exact value can be known.

- Other attributes can be used to fully define the possible action. These attributes can be different from one activity to another and also from one application to another.

Therefore, one can write an elementary action as:

$$
\alpha_{i}=\left\{d_{i, j}=\left(a_{j}, v_{i, j}\right)\right\}_{j=1}^{\operatorname{Card}\left(\alpha_{i}\right)}
$$

For every application, there is a countable set of elementary actions. Therefore the following notation is used:

- $\mathcal{A}$ : The countable set of elementary actions for a specific application.

- $\operatorname{Card}(\mathcal{A})$ : The number of elementary actions.
$\mathcal{A}$ is defined as:

$$
\mathcal{A}=\left\{\alpha_{i}\right\} \text { with } i \in\{1, \ldots, \operatorname{Card}(\mathcal{A})\}
$$

\subsection{Local Decision}

The local decision represents the elementary action execution on the system. A Local decisions $e_{i}$ is defined with a countable set of descriptors $d_{i, j}$ (attribute $a_{j}$, value $v_{i, j}$ ). First, local decision inherits the descriptors of the elementary action. They are completed by other descriptors that can be classified into three categories:

\section{- Schedule related descriptors:}

- Start time or date: To define the schedule of the actions, we need to define the start time or date of the decision.

- Allocated Resources: Some actions can require a special set of resources to be executed. Therefore, when these actions are transformed into decisions the required resources or the available resources are assigned to the local decision. The resources could be (i) material, like special tools for maintenance activity, or spare parts, or (ii) human, like a technician, maintenance teams, and production operators.

- Health descriptors: Here, we are going to define two attributes to describe the evolution of the system health state with the integration of the present decision. These attributes are:

- The initial health state indicators: This can be defined by a set of health indicators of the studied system before the execution of the local decision.

- The estimated final health state indicators: This is the set of the estimated final values of the health indicators after the execution of the local decision.

- Reliability level: This describes the reliability of the system when executing the specified local decision.

- RUL values, and so on.

- Application related descriptors: In this section of the local decision definition, we will define the attributes of the decision in the context of the proposed application. These attributes are used in the computation of the performance indicators of the application. For example, in this section we can find:

- Cost: The estimated cost of the execution of the action.

- Benefits: The likely benefits from the execution of the action.

- Mission success rate: The predicted success rate of the mission if the action defined is integrated.

- and so on.

So a local decision $e_{i}$ can be written as:

$$
e_{i}=\left\{d_{j}^{i}=\left(a_{j}, v_{i, j}\right)\right\} \text { with } j \in\left\{1,2, \ldots, \operatorname{Card}\left(e_{i}\right)\right\}
$$


We suppose that for any studied application there is a finite number of the local decisions, thus we note:

- $\mathcal{E}$ : The countable set of local decisions.

- $\operatorname{Card}(\mathcal{E})$ : The number of the local decisions in set $\mathcal{E}$.

Therefore, $\mathcal{E}$ is defined as:

$$
\mathcal{E}=\left\{e_{i}\right\} \text { with } i \in\{1,2, \ldots, \operatorname{Card}(\mathcal{E})\}
$$

For most of the applications, the set of feasible local decision is time-sensitive. The set of feasible local decisions at instant $t$ is defined as a subset of the set of possible decisions $\mathcal{E}$. The feasibility of local decisions is defined by the time constraints and the local prognosis of the elementary action they represent. This point is more detailed in section 4.1. One can note:

- $\mathcal{F}(t)$ : The countable set of feasible local decisions at instant $t$.

- $\operatorname{Card}(\mathcal{F}(t))$ : The number of feasible local decisions in set $\mathcal{F}(t)$

Therefore, $\mathcal{F}(t)$ is defined by:

$$
\mathcal{F}(t)=\left\{e_{i}\right\} \text { with } i \in\{1,2, \ldots, \operatorname{Card}(\mathcal{F}(t))\}
$$

With:

$$
\mathcal{F}(t) \subset \mathcal{E}
$$

\subsection{Global Decision}

A global decision is a sequence of local decisions over a duration $H$ called decision horizon. Therefore, a global decision $g_{u}(t)$ can be defined with a countable set of descriptors $D_{u, j}$ (attribute $A_{j}$, value $V_{u, j}$ ). The descriptors of the global decision can be classified into three categories:

- Composition Descriptors: In this set of descriptors, the composition of the global decision is defined. Thus it contains two attributes:

- The Sequence of local decisions: This attribute contains the sequence of local decisions. For example the schedule of jobs and maintenance actions.

- The Duration of the Horizon: This attribute will define the duration of the decision horizon of the global decision.

- Health Descriptors: Similar to the local decisions, global decision defines the estimated evolution of the system's health state. For this some of the most commonly used attributes are presented:

- Initial Health State Indicators: This contains the set of the initial health indicators before applying the global decision.

- Final Health State Indicators: This is the set of the estimated final health indicators after applying the global decision.
- Reliability Level: The evolution of the reliability of the system while applying a global decision, could be a relevant indicator of the system health.

$-\quad \ldots$

- Application Related Descriptors: In this category, one defines the attributes of the decision that are oriented to the application objective. These attributes are the performance indicators of the application plus other descriptors that can be used in the computation of the performance indicators. Some examples of the most common indicators are:

- The cost of the global decision

- The predicted gain of the global decision

- The Occupation (\%) of the system

$-\ldots$

Therefore, $g_{u}(t)$ is defined as:

$g_{u}(t)=\left\{D_{u, j}=\left(A_{j}, V_{u, j}\right)\right\}$ with $j \in\left\{1, \ldots, \operatorname{Card}\left(g_{u}(t)\right)\right\}$

To preserve the consistency of the definitions, the following notations related to the definition of the global decision are proposed. Global decisions are related to their time of construction, thus one can note:

- $\mathcal{G}(t)$ : The set of global decisions at time $t$.

- $g_{u}(t)$ : The $u^{t h}$ global decision at time $t$ of $\mathcal{G}(t)$.

- $\operatorname{Card}(\mathcal{G}(t))$ : The number of global decisions in $\mathcal{G}(t)$.

Therefore, $\mathcal{G}(t)$ is defined as:

$$
\mathcal{G}(t)=\left\{g_{u}(t)\right\} \text { with } u \in\{1,2, \ldots, \operatorname{Card}(\mathcal{G}(t))\}
$$

In some cases, the number of possible actions can be tremendous causing a considerable number of feasible combinations and consequently a big set of global decisions. This can be considered as an optimization problem. Therefore, a suitable optimization method from the literature should be used to construct a smaller set of global decisions by finding the most interesting sequences of elementary actions. The choice of such an optimization method is based on the application specifications in terms of constraints, real-time performances, the quality of the desired solution and so on. Such a point should be discussed during the implementation phase.

While the other descriptors may vary from one application to another, the composition descriptors are common for all types of applications since they are related to the proposed framework. Therefore descriptors $D_{u, 1}$ and $D_{u, 2}$ are defined as:

$$
D_{u, 1}=\left(\text { Sequence of local decisions }, V_{u, 1}\right)
$$

where:

$$
V_{u, 1}=\left(e_{i}\right)_{1}^{m_{g_{u}(t)}}
$$


and: $m_{g_{u}(t)}$ : The number of local decisions that build global decision $g_{u}(t)$

$$
D_{u, 2}=\left(\text { Decision Horizon, } V_{u, 2}\right)
$$

where:

$$
V_{u, 2}=H(t)
$$

$H(t)$ is the duration of the decision horizon at time $t$.

\section{PHM Framework Adaptation}

One aim of this work is to highlight the inter-dependencies of prognostics and decision-making. Thus, these two processes are integrated into a common process within the PHM context. The resulted process consists of building global decisions while iteratively estimating the outcomes of elementary actions through a decision-building loop. The decision building loop highlights the interaction between decision-making and prognostics by including the future loads and conditions in the prognostic module and emphasizing prognostic information in the optimization algorithm. Although the decision applying loop is a natural process, it was not presented as a component of the PHM framework. In this paper, we formalize the definition of the decision applying loop and we study its parameters. This loop estimates dynamically the remaining useful life and/or the health indicators of the system such as its reliability, degradation level and so on. Thus, it captures the effect of decisions on the system's health states. Finally, to update parameters of both short and long-term prognostics process, we introduce an information loop, which aims at comparing real evolution of the system health indicators to the estimated one during the previous period. Based on this comparison, if the real data is judged to be relevant (important deviation of the estimation) the prognostic method parameters should be updated. The upgrade of the PHM framework is presented in this section through the aforementioned loops.

\subsection{Estimators and Decisions Builders}

The proposed method to highlight the prognostic decision interactions consists of building elementary actions into sequences of decisions while evaluating the outcomes of these local decisions in terms of the system's health and the objective of the studied application. In this context, two new modules are proposed to be added to the phases of analysis and decision support as shown in Figure 4.

A decision builder is joined to the decision support phase of the Figure 2. This decision builder module can interact with the prognostic phase using an estimator module that is designed to forecast the degradation level of the system. The two modules are shown in Figure 4 and described as:

- Decision builder: This module is responsible for building the global decisions thanks to the set of available elementary actions $\mathcal{A}$. This module is part of the decision support phase of the PHM process. It has four main functions; (i) finding the possible elementary actions, and create the set of feasible local decisions $\mathcal{F}(t)$ (ii) creating the set of possible sequences of local decisions $\mathcal{S}(t)$ at time $t$ (iii) evaluating the elements of $\mathcal{S}(t)$ (iv) selecting and scheduling the suitable sequence of local decisions $s^{*}$ from $\mathcal{S}(t)$. The decision builder is the first element of decision-making in which it will assemble a set of global decision $\mathcal{G}(t)$ at time $t$. Since post-prognostic decision-making is considered as an optimization problem, decision builders can be classified as an optimization method. Various optimization methods can be modified to become a decision builder, such as the search algorithms $D^{*}, A^{*}, \ldots$, heuristics like the ant colony optimization, particle swarm optimization, and so on. The selection of the suitable decision building method is application related and should be addressed during the implementation phase.

- Estimator: This is a function that aims to forecast the degradation of the system under the new local decision. It evaluates the outcome in terms of health state (i.e. degradation, reliability, ...) of the studied system for each possible elementary action at time $t$. It is also equipped with a virtual system, that contains the future state of the system once a local decision is selected by the decision builder. This module is part of the analysis phase of the PHM process. Figure 5 shows an example of the output of the estimator. Estimator could be defined as a short term prognostic algorithm that can integrate future loads of the system. The choice of the used algorithm in this process can be subject to some constraints like the execution time and/or precision. The estimator has to evaluate the outcome of a large number of elementary actions and for some time-sensitive application, it could be more beneficial to use a swift prediction algorithm to reduce the estimation time. For applications that are not time-sensitive but that require a high level of precision, the estimator could be a replicate of the prognostic algorithm. Therefore, the estimator could be any model-based or data-driven prognostic algorithm. One can use various types of methods as estimators, for example, auto-regressive methods, linear regressionbased methods, particle filters, neural networks, etc... The novelty here is to include the future local decisions in the estimation of the system future state. However, we limit the choice of the estimator by the conditions that it should be a short-term prognostic approach and it should take future decisions as an input.

The feasibility of local decisions and their sequences is a two steps process:

- In the first step, the decision builder considers all possible local decisions at an instant $t$. These possible local decisions are, then, tested against their time con- 


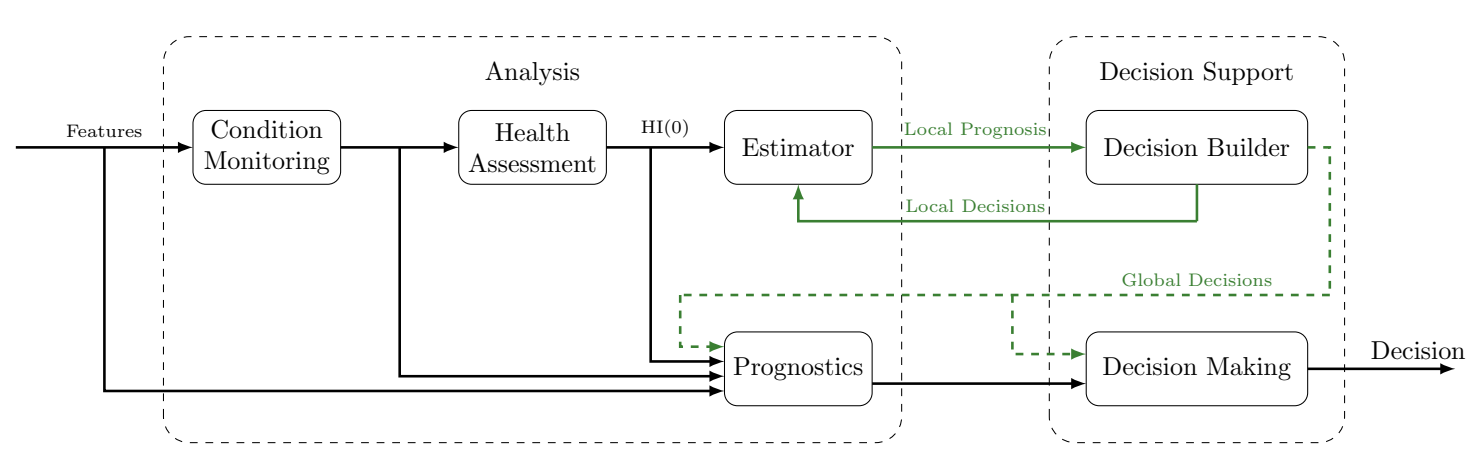

Figure 4. Block diagram of the decision building process

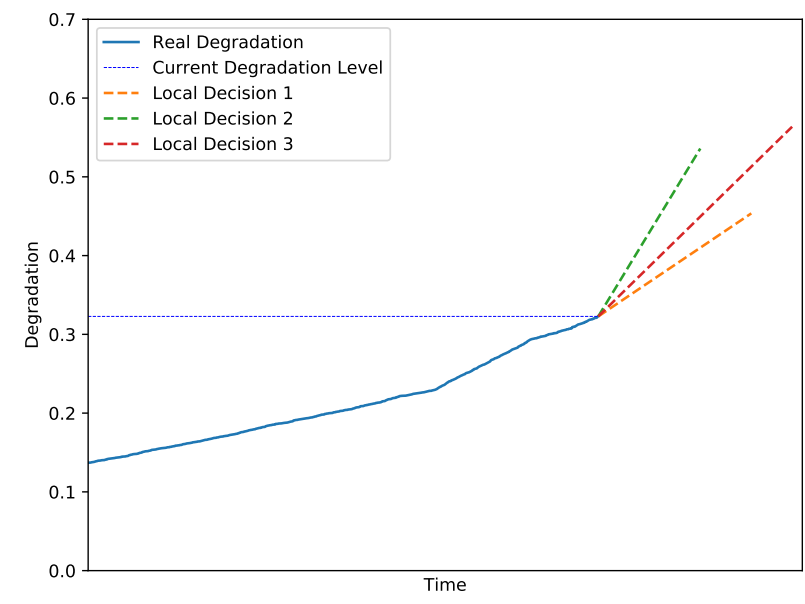

Figure 5. Example of the estimator output

straint. For example, let us consider the local decision of scheduling a production job at $t=100$ u.t. The job in question has a processing time of 50 u.t. meaning that its completion date will be equal to 150 u.t. But the job has a due date equal to 140 u.t. Thus it is judged as unfeasible. The time aspect of local decisions feasibility is presented in figure 6 . Where, $e_{1}$ is time-unfeasible and $e_{2}$ is time-feasible.

- The second step is dependent on the results of the local prognosis provided by the estimator. For example, if we consider another production job that is feasible from a time point of view. The job could be considered as unfeasible if the outcome of its local prognosis exceeds the failure threshold of the system. The health aspect of local decisions feasibility is presented in figure 7 . Where, $e_{1}$ is health-unfeasible and $e_{2}$ is health-feasible.

A sequence of local decisions is judged to be feasible if all the local decisions it contains are feasible in both time and health aspects.

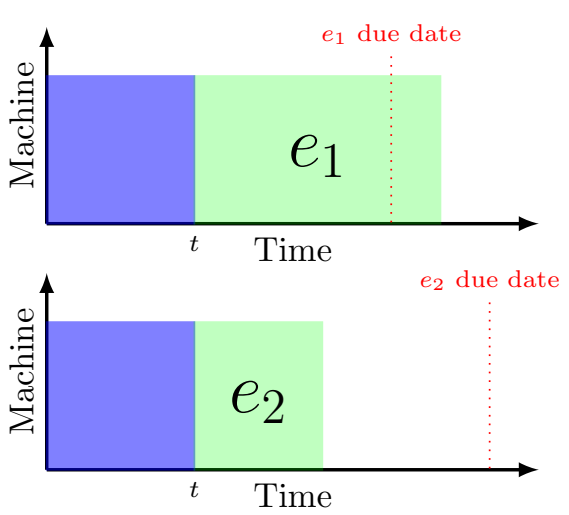

Figure 6. Time Feasibility of Local Decisions

\subsection{Decision Building Loop}

Figure 8 presents the sequential diagram of the decision building procedure. At the beginning of a PHM Stage $k$, the current state of the system is used to update the state of the estimator. A step of construction is initiated and the information is transmitted to the decision builder. The latter considers the set of elementary actions $\mathcal{A}$ and create the set of candidate local decision sequences $\mathcal{S}(t)$. The set is feed to the estimator to obtain the short term prediction of the systems' state under each of these sequences. Once all the local prognostics are performed the set of possible sequences is updated with the final estimated state of the system and returned to the decision builder. The latter selects one of the sequences $s^{*}$ based on the performance evaluation. The selected sequence $s^{*}$ is given to the estimator to re-update its state. The estimator, then, checks the remaining time to the end of the decision horizon. If the duration of the decision horizon is reached, the building process is completed. Else, another step of construction is launched, until the end of the decision horizon is reached.

Once the decision-making process has received the set of global decisions $\mathcal{G}(t)$. The process can evaluate the evolution 


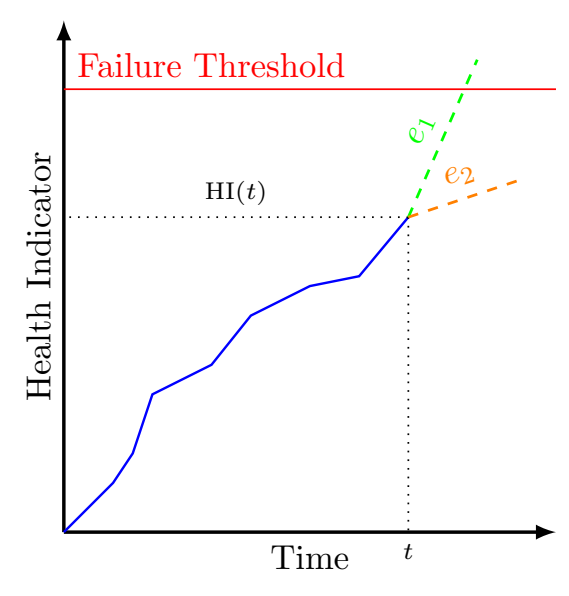

Figure 7. Health Feasibility of Local Decisions

of the health indicators of each global decision by running it through the prognostic process then select the suitable global decision $g^{s}(t)$ to be applied on the system or it can go directly to the selection process.

Although the construction of one global decision is quite a sequential process, the building of the set of global decisions $\mathcal{G}(t)$ can be done concurrently. The possibility of constructing several global decisions at the same time depends on the capacity of the chosen algorithms for the decision builder and the estimator. For example, if one chooses the ant colony optimization as decision builder and linear regression as an estimator, the construction of $\operatorname{Card}(\mathcal{G}(t))$ global decision can be done all at once.

\subsection{Decision Applying Loop}

As said earlier, decisions are built in the PHM context to prevent a system from failing or to mitigate the effects of failure if avoiding it is no longer an option. Thus, when a global decision is selected, it will be applied in totality or partially on the system. The application of decisions (partial or global) leads to a real evolution of the health state of the system that can differ from the estimated one. Moreover, to capture the effect of this feedback, a re-execution of the prognostic and decision process is needed. Therefore, the proposed framework is set to be executed more than once, at specified moments called stages.

At a specific stage $k$ (when $t=T_{k}$ ), the system undergoes a normal PHM process, from data acquisition to health indicators assessment. Once the current state of the system is identified, the decision building process is triggered and the construction of global decisions over the specified horizon begins. When the decisions are built, the decision-making module selects a suitable decision for the next horizon. The selected decision $g_{k}^{s}=g^{s}\left(T_{k}\right)$ is applied to the system until
$t=T_{k+1}$ the time of the next stage $k+1$. At this new stage $k+1$, the decision horizon is shifted, and the system undergoes once more the same PHM process. This process can be done over and over again as much as needed. This dynamic is represented in Figure 9 by the red feedback wire. An example of the decision process at stages $k$ and $k+1$ is shown in Figure 10.

The applied part of the selected global decision $g_{k}^{s}$ between two consecutive stages $k$ and $k+1$ is called partial decision of stage $k$ (denoted $p_{k}$ ). This partial decision is also represented by the set of descriptors $\left(D_{u, j}\right)$ like the global decision, although, the values $\left(V_{u, j}\right)$ of the attributes $\left(A_{j}\right)$, here, are the result of the application of the global decision on the system. Thus these values $V_{u, j}$ represent the real values of the state of health evolution, the cost, and the duration.

The partial decision duration or the duration between two stages of decision is an important parameter of the proposed framework. If this duration is reduced to be equal to the duration of a local decision, then the proposed framework is similar to the activities of real-time decision-making like in the case proposed by Zhang in (Zhang et al., 2014), or the automatic control decisions detailed by Pereira et al. in (Pereira et al., 2010), Nguyen et al. (Nguyen, Dieulle, \& Grall, 2014) or by Vieira et al. in (Vieira, Kawakami, Galvao, \& Yoneyama, 2015) in which the dynamic of the RUL estimation is high. On the Opposite, if the duration of the partial decision is extended to be equal to the decision horizon duration, here the proposed framework is similar to the works that integrates a rolling horizon like the work of Van Horenbeek and Pintelon (Van Horenbeek \& Pintelon, 2013), plus if we suppose that the PHM process will be executed only once, the framework will be treating the post-prognostic decision-making like the one presented by Herr et al. in (Herr et al., 2014) or (Herr et al., 2017).

The decision horizon and the duration of the partial decision are two application-related parameters. We do not define a formula for these two parameters here since they highly depend on the application. When applying this framework, one has to study their suitable values to optimize the decision process.

\subsection{Information Loop}

When the global decision is built, the evolution of the system health indicators is estimated under the selected local decisions. On the other hand, when the partial decision is applied to the system, the real evolution of the system health indicators is acquired and identified. Therefore, the partial decision contains both the estimated evolution and the real one. The difference between the estimated and the resulted evolution of the system health indicators provides the errors of local prognostics that can be measured by different indicators like the root mean square error (RMSE). An example of the real and 


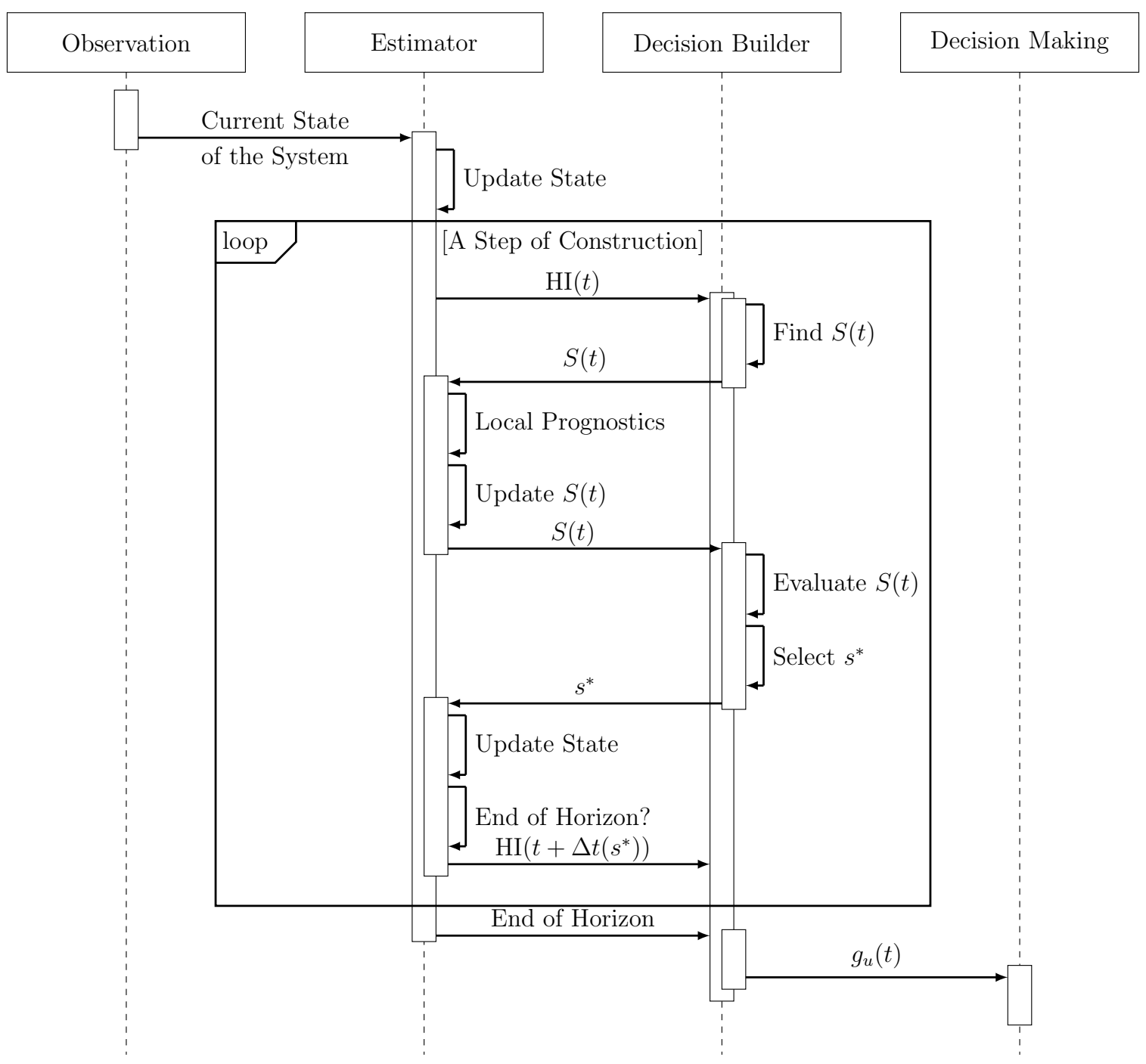

Figure 8. The Decision Building Sequential Diagram

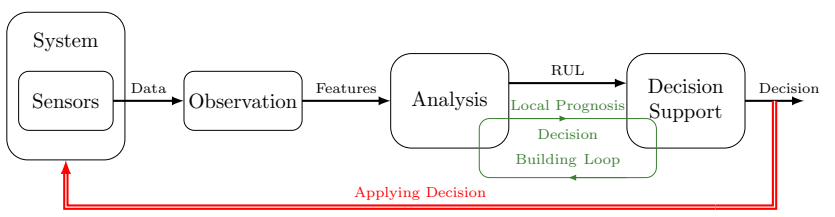

Figure 9. The Decision Applying Loop

estimated evolution of a component are shown in Figure 12. The evolution of the corresponding RMSE through the stages of the example is shown in Figure 13. Based on these errors or their representative metrics, one can fine-tune the parameters of the estimators to improve the accuracy of the local prognostics. This allows to increase the efficiency of the decision process. Thus the third loop of the proposed framework is defined as information loop as presented in Figure 11.

The information loop is considered as a foundation for methods that aims at fine-tuning the parameters of the estimator. While the role of the information loop is to gather the real evolution of the system state and compare it to the estimated one. The resulting error can be directly used to adjust the parameters of the estimator. One can find several methods for the estimator that benefits from the implementation of the information loop such as artificial intelligent methods, regression methods, case-based reasoning, and so on. For instance, the newly available degradation data can be added to the set of training data for artificial intelligence-based estimators (like neural networks). Another example is the use of a case-based reasoning approach as an estimator. In this case, one can imagine the importance of the feedback information provided 


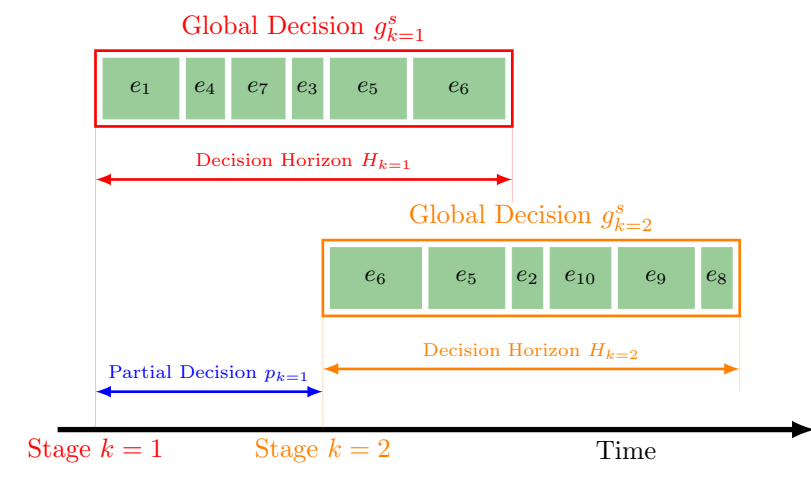

Figure 10. Illustration of the results of the decision applying loop

by the information loop, in the phase of revision to improve the estimator's parameters and/on in the phase of memorizing to save new relevant cases. In the case of regression methods, the new data can be used to re-adapt the parameters or even the type of regression model.

The integration of such a loop into a PHM process keeps the systems model up to date with the real system. Thus for a complex system with high variability of operating conditions or system behavior, such an information loop has an important in monitoring the system and in allowing the adaptation of the used methods to the newly available conditions.

\subsection{Overview of the Proposed Framework}

To summarize this subsection, a new PHM process framework is proposed in which improvements are made on the classic PHM process by integrating three closed-loops processes (Figure 14). This new framework aims at dynamically estimating the RUL of the system while integrating the feedback of the decision on the system through the decision applying loop. The presented framework emphasizes the relationship between decision-making and prognostic modules by building decisions and estimating their outcomes via the decision building loop. Moreover, the information loop can be used to optimize the estimator's parameters and to analyze its performance.

To guarantee the efficiency of this framework, one should choose the suitable methods to use in the different modules. Some of the possible methods have been discussed earlier in the definition of the different modules. Yet, the choice of methods needs to be based on the treated problem and the requirement of the application in terms of execution time, accuracy and other constraints metrics.

In the proposed framework, local and global decisions are defined as a set of descriptors. However, not all descriptors influence the space of possible decisions. For example, the id of the production order can be a descriptor of the solution space while the cost, benefits, and the machine's health state at the start and the end of the decision are a consequence of the schedule/decision. The space of descriptors for local and global decisions is incredibly large. Therefore, when applying this framework on a real-life application, a much more detailed investigation should take place to identify the adequate descriptors for the intended application. Furthermore, in a general sense, a descriptor could have a large space of possible values. This could result in a computational explosion. However, in a practical scenario, the options for each descriptor have only a few options available. These options could be derived from previous experiences and/or from the machine's design.

\section{Case Study Illustration}

Considering the previously presented framework, we propose now to illustrate how it can be used on an application. All the details about this illustration are given in the paper of Bougacha et al. (Bougacha, Varnier, Zerhouni, \& HajriGabouj, 2018). The section contains first a definition of the problem then some elements of resolution according to the proposed framework.

\subsection{Problem Description}

The proposed application is inspired by the studies done on the computer numerical control (CNC) machines used for machining. The considered system is known to operate on different products that come in a variety of sizes, shapes, and materials. Thus, the machining operations are different from one product to another. Consequently, the degradation of the system is highly influenced by the type of product that is processed. The CNC machines, also, provide a range of possible operating parameters. These parameters are defined as the cutting speed and the feed rate. The degradation of the system is assumed to be influenced by the chosen production profile (i.e. the cutting speed and the feed rate). In this application, without any loss of generality, it is assumed that the higher the speed of cut and the higher the feed rate is, the faster the components are deteriorating.

Let's consider a single CNC machine composed of $n$ nonidentical components that are subject to wear and tear. The failure of component $i$ causes the failure of the whole system (i.e. n-component series system). The failure of a component and/or the system is noticed immediately without inspection. The degradation of the components is influenced by the type of product $\rho_{p}$ and the operational profile $\sigma_{j}$ used. The component degradation is described through a variable $D_{i}$ with $i \in\{1, \ldots, n\}$, where $\left\{D_{i}(t), t \geq 0\right\}$ is a homogeneous Gamma Process with shape parameter $\nu_{i}(t)$ and scale parameter $\mu_{i}$ and the following properties:

- $D_{i}\left(t^{\prime}=0\right)=0$

- $D_{i}(t)$ has independent increments 


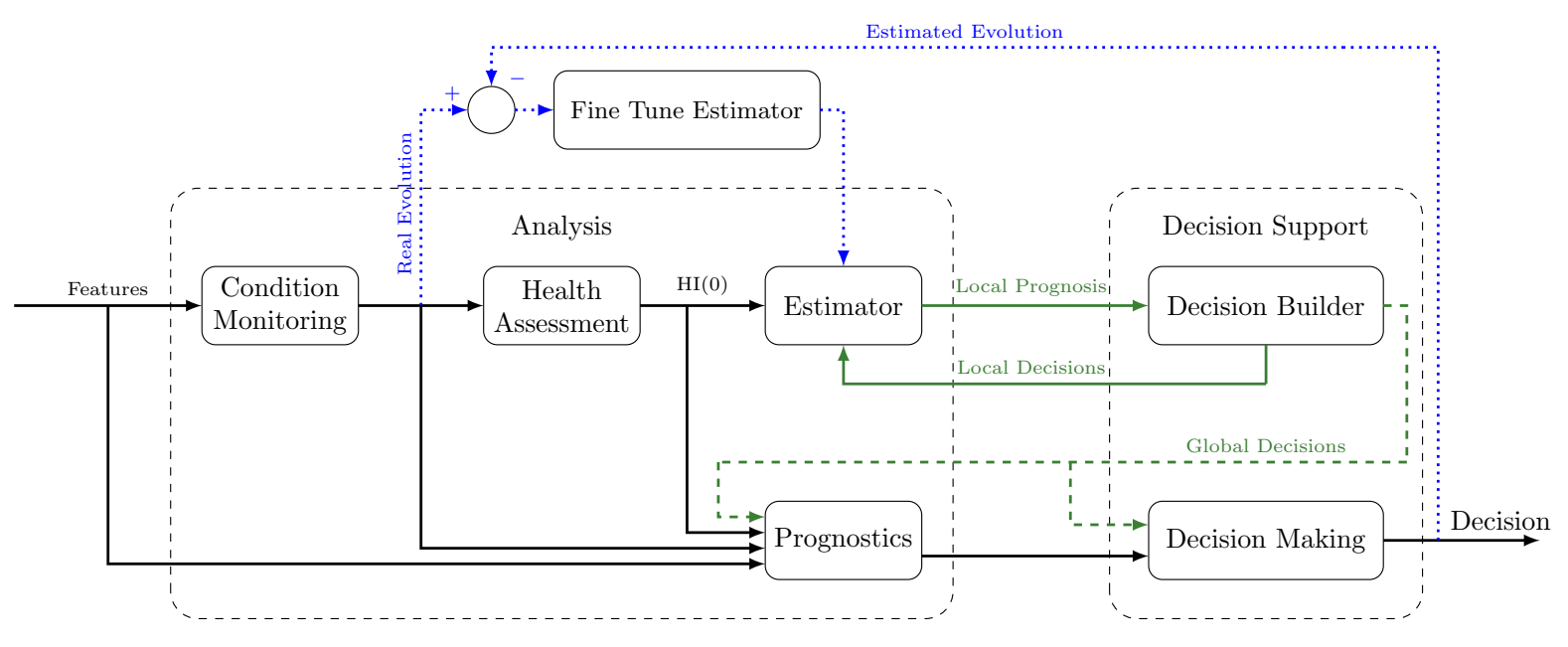

Figure 11. Information Loop

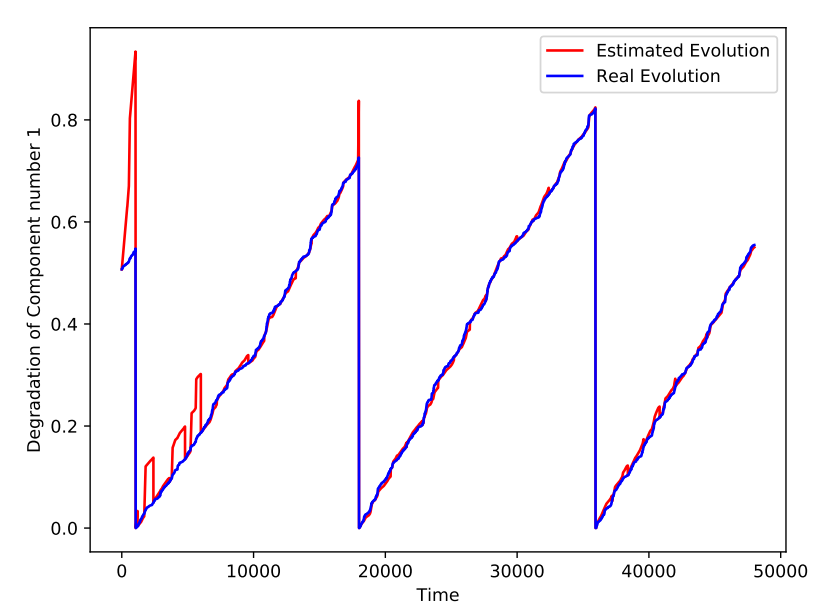

Figure 12. Degradation Evolution of Component Number 1

- For $t>0$ and $h>0$ during which the CNC machine is producing product type $l$ with profile $j, D_{i}(t+h)-$ $D_{i}(t)$ follows a gamma distribution with shape parame$\operatorname{ter}\left(\nu_{i}(t+h)-\nu_{i}(t)\right) * \gamma_{j}$ and scale parameter $\rho_{p} * \mu_{i}$

The following assumptions are verified in this example:

- The degradation processes of the components are independent. In other words, we only considered structural dependencies from the dependencies described by Nicolai and Dekker (Nicolai \& Dekker, 2008).

- When the degradation $D_{i}$ of component $i$ reaches the failure threshold set at $1\left(D_{i} \geq 1\right)$, the component fails.

- The degradation level of the components is retrieved at each inspection date.

- The inspection dates are equally distant and defined by the value of the duration of the Partial Decision.

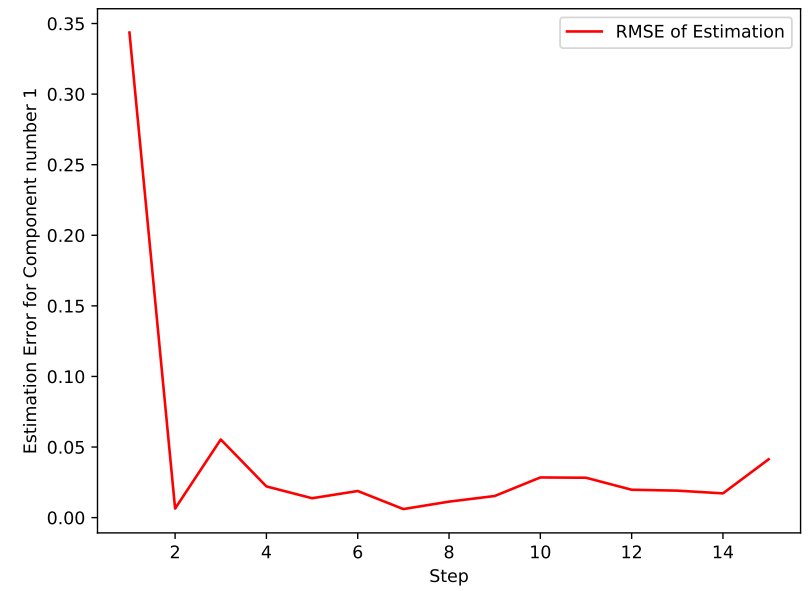

Figure 13. Evolution of RMSE of Component Number 1

- The Partial Decision is equal to the Decision Horizon.

- The inspections are performed at zero cost.

- Maintenance interventions bring the component to an 'as good as new' status with a degradation level equal to zero.

- The corrective maintenance cost and duration are higher than the predictive ones.

- $P$ types of products can be produced by the $\mathrm{CNC}$, each type $p$ has a production cost per unit per production profile $C_{p, j}$ and a price per unit $S P_{p}$.

- $J$ production profiles can be performed by the CNC, each production profile has a production duration per unit per type of product $P_{j, p}$.

The aim is to better manage the machine in terms of availability and reliability. Thus, a compromise between producing different orders and maintaining the system is needed to 


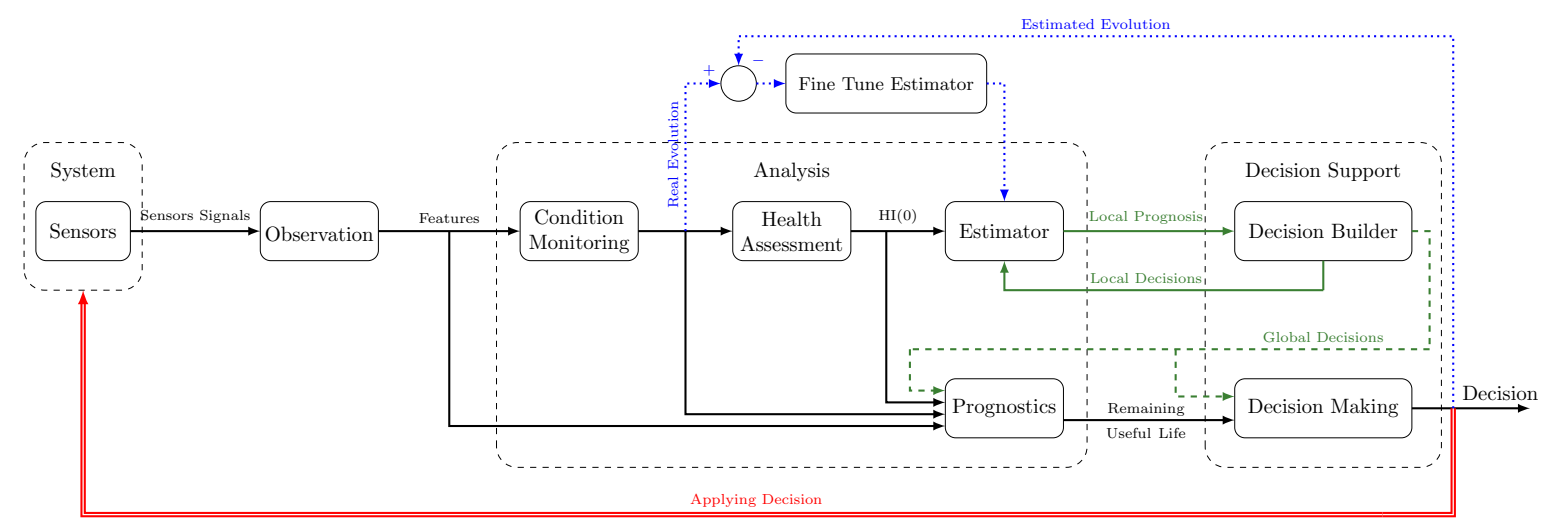

Figure 14. The Closed Loops PHM Process

maximize the benefits of the workshop. The benefits of the workshop are defined by the difference between the total cost over the simulation horizon (including production cost, maintenance cost and the cost of lost opportunity when the machine is capable of producing but no order is possible) and the gain obtained by selling the produced orders.

The presented problem treats mixed decision-making, in which two options are available either produce a job or schedule a maintenance intervention. Jobs in this context are defined as in the case of production scheduling problems, i.e. each production order $l$ requires a quantity $q_{l}$ of product $p$, the delivery of the order should be done before a due date $d_{l}$ otherwise some penalties are to be paid for delays, and once the time exceeds the deadline $D L_{l}$ the order is no longer valid.

In the next subsection, some elements of resolution according to the proposed framework are presented. First, the elementary actions are defined. Then, an example of local decisions and global decisions is given.

\subsection{Elements of Resolution}

\subsubsection{Elementary Actions}

To solve the aforementioned problem, one starts by defining possible elementary actions. Since the problem is treating mixed decisions, two kinds of elementary actions are involved:

- Operational Actions; The action should specify the order to produce and with which production profile.

- Maintenance Actions; The action points out the component(s) to be maintained to improve the overall reliability of the machine.

These elementary actions can be defined as in tables 1, 2 .
Table 1. An Example of an Operational Elementary Actions

\begin{tabular}{|c||c|}
\hline$a_{i}$ & $v_{i, j}$ \\
\hline Type & Production Order \\
\hline Target & CNC Machine \\
\hline Task & Order number 2 \\
\hline Cutting Speed & 100 rpm \\
\hline Feed Rate & 10 products per hour \\
\hline Duration & 48 minutes \\
\hline
\end{tabular}

Table 2. An Example of a Maintenance Elementary Actions

\begin{tabular}{|c||c|}
\hline$a_{i}$ & $v_{i, j}$ \\
\hline Type & Maintenance \\
\hline Target & Component C1 \\
\hline Duration & 60 minutes \\
\hline
\end{tabular}

\subsubsection{Local Decisions}

As defined earlier, local decisions are obtained by sche-duling the elementary actions in the process of jointly scheduling production and maintenance at time $t$. Thus, the elementary actions presented in tables 1 and 2, are transformed into the following local decisions, presented in tables 3 and 4 .

\subsubsection{Global Decisions}

Global decisions are built in the decision building loop by scheduling local decision in a sequence. As defined earlier, global decisions present different categories of descriptors but they all have in common the first two descriptors; which define the sequence of the local decisions and the decision horizon. An illustration of a global decision is given in table 5 .

\subsection{Numerical Example}

Let us consider the numerical example in which the machine is composed of three stochastically degrading components. The purpose of this example is to provide proof of the utility of such a proposed framework. 
Table 3. An Example of an Operational Local Decision

\begin{tabular}{|c|c|c|}
\hline $\begin{array}{c}\text { Set of } \\
\text { Descriptors }\end{array}$ & Attribute & Value \\
\hline $\begin{array}{c}\text { Action } \\
\text { Descriptors }\end{array}$ & $\begin{array}{c}\text { Type } \\
\text { Target } \\
\text { Task } \\
\text { Cutting Speed } \\
\text { Feed Rate } \\
\text { Duration }\end{array}$ & $\begin{array}{c}\text { Production Order } \\
\text { CNC Machine } \\
\text { Order number } 2 \\
100 \mathrm{rpm} \\
10 \text { products per } \\
\text { hour } \\
48 \text { minutes }\end{array}$ \\
\hline $\begin{array}{l}\text { Resolution } \\
\text { Descriptors }\end{array}$ & $\begin{array}{l}\text { Start Time } \\
\text { Needed } \\
\text { Resources }\end{array}$ & $\begin{array}{c}t=200 \\
\text { none }\end{array}$ \\
\hline $\begin{array}{c}\text { Health } \\
\text { Descriptors }\end{array}$ & $\begin{array}{c}\text { Initial } \\
\text { Degradation } \\
\text { Final } \\
\text { Degradation } \\
\text { Reliability } \\
\text { Level }\end{array}$ & $\begin{array}{c}(0.547, \ldots, 0.012) \\
(0.597, \ldots, 0.147) \\
0.4\end{array}$ \\
\hline $\begin{array}{l}\text { Application } \\
\text { Related } \\
\text { Descriptors }\end{array}$ & $\begin{array}{c}\text { Quantity } \\
\text { Produced } \\
\text { Cost }(€) \\
\text { Gain(€) } \\
\text { Utilization(\%) } \\
\text { Maintenance }\end{array}$ & $\begin{array}{c}120 \\
\\
12 \\
20 \\
20 \% \\
\text { none }\end{array}$ \\
\hline
\end{tabular}

In this aim, the proposed framework is using an ant colony optimization (Dorigo \& Caro, 1999) method as a decision builder module and a simple linear regression for the estimator. The components degradation data is obtained by simulating the gamma process for different combinations of production profiles and products. then, based on which the parameters of the linear regression are obtained for each component under different operating conditions. New degradation data is progressively acquired when we simulate the application of new decisions on the machine. This new data will be added to the estimator to fine-tune its parameters and thus improving its accuracy as it is shown in Figures 12 and 13.

The proposed approach will be referenced as PHM+. The system is simulated on a small simulation horizon. The obtained results are compared to a classical method of production scheduling in literature combined with a periodicinspection condition-based maintenance protocol. This approach will be noted M-CBM. For more detail on the MCBM algorithm and the ant colony algorithm used, one can refer to the following works (Bougacha et al., 2018). As a small description, the algorithm used for production scheduling is the Moore Algorithm as defined in (Moore, 1968), the choice of this algorithm is based on the objective of minimizing the production cost by minimizing the number of tardy jobs. The CBM protocol consists of periodically inspect the machine, if the degradation of one or more components exceeds a maintenance threshold, a preventive replacement
Table 4. An Example of a Maintenance Local Decision

\begin{tabular}{|c|c|c|}
\hline $\begin{array}{c}\text { Set of } \\
\text { Descriptors }\end{array}$ & Attribute & Value \\
\hline $\begin{array}{c}\text { Action } \\
\text { Descriptors }\end{array}$ & $\begin{array}{c}\text { Type } \\
\text { Target } \\
\text { Duration }\end{array}$ & $\begin{array}{l}\text { Maintenance } \\
\mathrm{C} 1 \\
60 \text { minutes }\end{array}$ \\
\hline $\begin{array}{l}\text { Resolution } \\
\text { Descriptors }\end{array}$ & $\begin{array}{l}\text { Start Time } \\
\text { Needed } \\
\text { Resources }\end{array}$ & $\begin{array}{c}t=400 \\
\text { M-Operator } 2\end{array}$ \\
\hline $\begin{array}{l}\text { Health } \\
\text { Descriptors }\end{array}$ & $\begin{array}{c}\text { Initial } \\
\text { Degradation } \\
\text { Final } \\
\text { Degradation } \\
\text { Reliability } \\
\text { Level } \\
\end{array}$ & $\begin{array}{c}(0.897, \ldots, 0.562) \\
(0.0, \ldots, 0.562) \\
0.8\end{array}$ \\
\hline $\begin{array}{l}\text { Application } \\
\text { Related } \\
\text { Descriptors }\end{array}$ & $\begin{array}{c}\text { Quantity } \\
\text { Produced } \\
\text { Cost }(€) \\
\text { Gain(€) } \\
\text { Utilization(\%) } \\
\text { Maintenance }\end{array}$ & $\begin{array}{c}0 \\
\\
20 \\
0 \\
0 \% \\
2\end{array}$ \\
\hline
\end{tabular}

takes place otherwise the machine continue its normal function. While functioning, and if the degradation of one or more components exceeds a failure threshold, the machine is stopped and corrective maintenance takes place. Therefore, the combination of Moore algorithm and CBM strategy aims to minimize the total cost of the solution (i.e. maintenance and production costs). Corrective maintenance is assumed to be more costly and takes more time than a preventive intervention.

Both PHM+ and M-CBM are simulated in the same period and the evolution of the component's degradation under the two methods are presented respectively in Figures 15 and 16. It is important to note that the dashed red line presents the failure threshold. Once the degradation of a component exceeds this threshold the component breaks down and corrective maintenance takes place. One can easily note that the degradation of the components is better managed with $\mathrm{PHM}+$, this can be deduced from the number of maintenance activities processed on the different components.

Another comparison of the performances of the two methods is presented in table 6 . The methods are compared in terms of:

- Total cost: including production cost, maintenance cost, inspection cost, and unavailability cost.

- Expected benefits of the workshop.

- The number of corrective maintenance.

- The number of pieces produced during the simulation.

As shown in table 6, the cost generated using PHM+ is lower than the one obtained by the M-CBM. This can be explained 
Table 5. An Illustration of a Global Decision

\begin{tabular}{|c|c|c|}
\hline $\begin{array}{c}\text { Set of } \\
\text { Descriptors }\end{array}$ & Attribute & Value \\
\hline $\begin{array}{c}\text { Composition } \\
\text { Descriptors }\end{array}$ & $\begin{array}{c}\text { Sequence }\left(e_{i}\right) \\
\text { Decision } \\
\text { Horizon }\end{array}$ & $\begin{array}{c}\left(e_{1}, \ldots, e_{m_{g_{k, u}}}\right) \\
H_{k}\end{array}$ \\
\hline Health & Initial & $(0.897, \ldots, 0.562)$ \\
Descriptors & Degradation & \\
& Final \\
& Degradation & $(0.190, \ldots, 0.762)$ \\
Application & Quantity & 1200 \\
Related & Produced & 260 \\
Descriptors & Gast $(€)$ & 490 \\
& Gain( $(€)$ & $98 \%$ \\
& Utilization( $(\%)$ & 2 \\
& Maintenance & \\
\hline
\end{tabular}
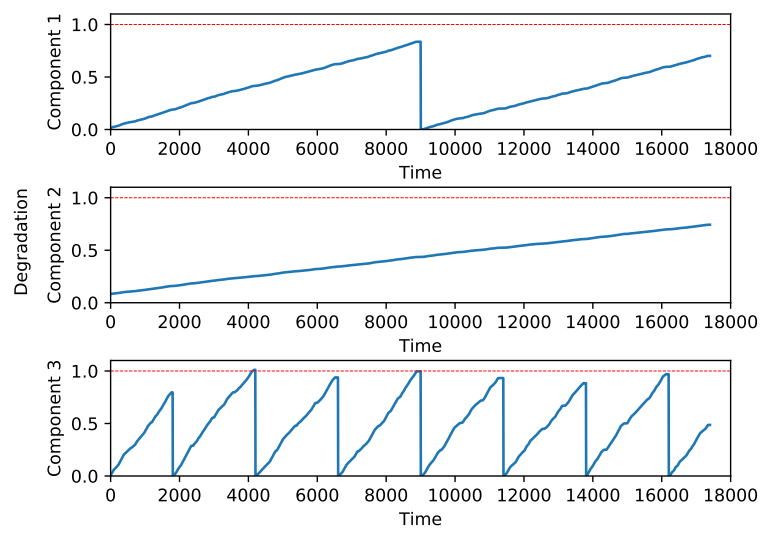

Figure 16. M-CBM Results

Table 6. Results Comparison
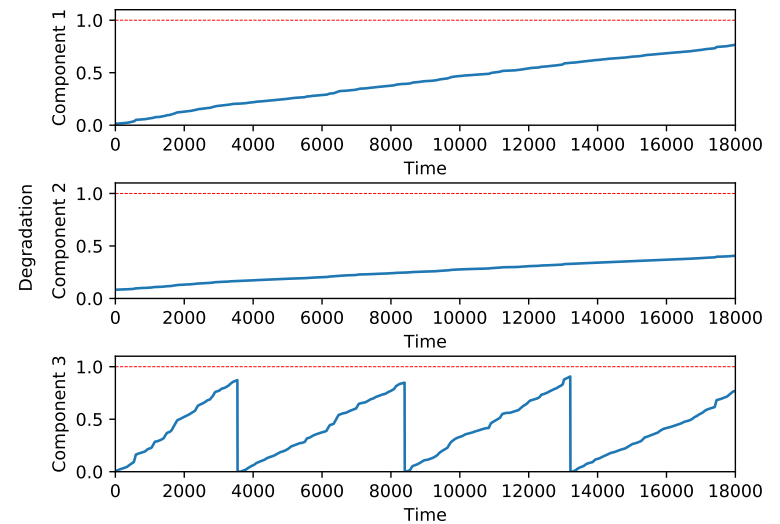

Figure 15. PHM+ Results

by the fact, that PHM+ does not present any corrective interventions and it uses different production profiles (rotation speed and feed rate) that in some cases have a lower cost than the profile used by the M-CBM. M-CBM presents two correctives maintenance, these actions can be seen in figure 16 , on component number 3 at times 4158 and 9200. Although the quantity of pieces produced by $\mathrm{M}-\mathrm{CBM}$ is larger than the one produced by $\mathrm{PHM}+$, the expected benefits of PHM+ is higher. The ant colony optimization used in the decision building loop of PHM+ aims to maximize the benefits of the workshop by:

- Reducing maintenance interventions.

- Prioritizing jobs that produce products with higher revenue.

- Minimizing the wasted time when the machine is available for producing but no order is available for production by using a slower production speed.

\begin{tabular}{|c||c|c|}
\hline Criteria & PHM+ & M-CBM \\
\hline Total Cost $(€)$ & 13268,86 & 17114,58 \\
\hline Benefits $(€)$ & 19444.4 & 16986.02 \\
\hline $\begin{array}{c}\text { Number of Corrective } \\
\text { Maintenance }\end{array}$ & 0 & 2 \\
\hline Quantity Produced & 14591 & 15624 \\
\hline
\end{tabular}

One can conclude that the use of the proposed PHM framework is, potentially, beneficial compared to the classical ways of decision-making for predictive maintenance. Therefore, the integration of prognostics and decision-making in a common process can improve both the prognostics performance and the health management of the considered system.

\section{Conclusion}

In this paper, we presented an adaptation of the existing PHM framework by integrating Prognostics and decision-making in a common process. This new process presents an enhancement for the decision-making of the previous process that has been introduced by the OSA-CBM (Lebold \& Thurston, 2001). The main idea of the updated framework is to build decisions from elementary actions. At each step, the decision builder considers the system's health status and the possible elementary actions and builds a sequence of these actions. The obtained sequences are evaluated with a short term prognostic algorithm to estimate their impact on the system. This decision-building loop is based on the inter-dependencies between decision-making and prognostics. Thus by implementing this loop, the first objective, of emphasizing the relationship between decision-making and prognostics, is achieved. The built decisions are transferred to the decision-making and the prognostic modules to evaluate the evolution of the system under these global decisions. One global decision is then selected by the decision-making algorithm and applied to the system for a predefined duration. This loop of the 
decision application is responsible for the integration of the decision's effects on the system. Combined with the use of rolling decision horizons this allows us to update the RUL values and to integrate the new available prognostics information in the decision-making process iteratively. Therefore, the RUL of the system is dynamically estimated and this information is more explicitly integrated into the decision-making process. Finally, an information loop allows the adaptation of the estimator parameters from one stage to another. Thus, the framework can dynamically take into consideration the new changes in the system states and reducing the uncertainties that can be caused by the operating conditions (i.e. future loads and environmental context). An example of implementing this framework is given on a multi-component single machine. The machine is used for machining products. The considered example deals with joint optimization of production and maintenance.

As a perspective, new applications of the framework on more complex contexts are in progress. Multiple machines with different dependencies are in an ongoing study. On the other hand, the proposed framework requires further investigation of the possible methods to be used as estimators, decision builders, and decision-makers. For an instant, one potential idea could be studying the implementation of a modified case-based reasoning as both estimator and decision builder. Another possibility is to study the use of Multi-Agent Systems to obtain a distributed decision-making process to reduce the execution time. One can imagine different architectures for multi-agent systems in PHM, in which the agent could presents the resources (like machines, production operators, and maintenance teams) or the different modules of the proposed framework. The use of multi-agent systems would potentially reduce the complexity of the problem resolution.

\section{REFERENCES}

An, D., Kim, N. H., \& Choi, J.-H. (2015). Practical options for selecting data-driven or physics-based prognostics algorithms with reviews. Reliability Engineering \& System Safety, 133, 223-236.

Balaban, E., \& Alonso, J. J. (2012). An approach to prognostic decision-making in the aerospace domain (Tech. Rep.). National Aeronautics and Space Administration MOFFETT Field CA AMES Research Centre.

Bougacha, O., Varnier, C., Zerhouni, N., \& Hajri-Gabouj, S. (2018, dec). A post-prognostic decision approach for production and maintenance planning. In 48th conference on computers \& industrial engineering (2018).

Byington, C. S., Roemer, M. J., \& Galie, T. (2002). Prognostic enhancements to diagnostic systems for improved condition-based maintenance. In Proceedings, ieee aerospace conference, 2002. IEEE.

Cai, J., Li, X., \& Chen, X. (2016). Optimization of aeroengine shop visit decisions based on remaining useful life and stochastic repair time. Mathematical Problems in Engineering, 2016, 1-11.

Camci, F. (2009, September). System maintenance scheduling with prognostics information using genetic algorithm. IEEE Transactions on Reliability, 58(3), 539552.

Cheng, G. Q., Zhou, B. H., \& Li, L. (2018, July). Integrated production, quality control and condition-based maintenance for imperfect production systems. Reliability Engineering \& System Safety, 175, 251-264.

Cui, Y., Shi, J., \& Wang, Z. (2015, September). Discrete event logistics systems (dels) simulation modeling incorporating two-step remaining useful life (rul) estimation. Computers in Industry, 72, 68-81.

Daigle, M., \& Goebel, K. (2010). improving computational efficiency of prediction in model-based prognostics using the unscented transform. Annual Conference of the Prognostics and Health Management Society 2010.

De Medeiros, I. P., Rodrigues, L. R., Kern, C. S., dos Santos, R. D. C., \& Shiguemori, E. H. (2015). Integrated task assignment and maintenance recommendation based on system architecture and phm information for uavs. In 9th annual ieee international systems conference (syscon), 2015 (pp. 182-188). IEEE.

De Medeiros, I. P., Rodrigues, L. R., Santos, R., Shiguemori, E. H., \& Júnior, C. L. N. (2014). Phm-based multi-uav task assignment. In 8 th annual ieee systems conference (syscon), 2014 (pp. 42-49). IEEE.

Do, P., Voisin, A., Levrat, E., \& Iung, B. (2015). A proactive condition-based maintenance strategy with both perfect and imperfect maintenance actions. Reliability Engineering \& System Safety, 133, 22-32.

Dong, H., Jin, X., Lou, Y., \& Wang, C. (2014). Lithium-ion battery state of health monitoring and remaining useful life prediction based on support vector regressionparticle filter. Journal of power sources, 271, 114-123.

Dorigo, M., \& Caro, G. D. (1999, July). Ant colony optimization: a new meta-heuristic. Proceedings of the 1999 Congress on Evolutionary Computation.

Fitouri, C., Fnaiech, N., Varnier, C., Fnaiech, F., \& Zerhouni, N. (2016). A decison-making approach for job shop scheduling with job depending degradation and predic- 
tive maintenance. In $8 h$ ifac conference on manufacturing modelling, management and control mim (pp. 1490-1495). Troyes, France.

Frost, S., Goebel, K., Frost, A., Trinh, K., \& Balas, M. (2013). Integrating systems health management with adaptive controls for a utility-scale wind turbine. In 51st aiaa aerospace sciences meeting including the new horizons forum and aerospace exposition (p. 314).

Gertler, J. (2013). Fault detection and diagnosis. In Encyclopedia of systems and control (pp. 1-7). Springer London.

Goebel, K., Celaya, J., Sankararaman, S., Roychoudhury, I., Daigle, M. J., \& Saxena, A. S. a. G. G. R. A. (2017). Prognostics: The science of making predictions. CreateSpace Independent Publishing Platform.

Goebel, K., Iyer, N., \& Bonissone, P. (2006, March). Framework for post-prognostic decision support. Aerospace Conference, 2006 IEEE.

Gouriveau, R., Medjaher, K., \& Noureddine, Z. (2016). From prognostics and health systems management to predictive maintenance 1. John Wiley \& Sons.

Herr, N. (2015). Post-prognostic scheduling of heterogeneous distributed platforms (Unpublished doctoral dissertation). Franche-Comté University.

Herr, N., Nicod, J.-M., \& Varnier, C. (2014). Prognosticsbased scheduling in a distributed platform: Model, complexity and resolution. In Case, 2014 ieee international conference on automation science and engineering (pp. 1054-1059). IEEE.

Herr, N., Nicod, J.-M., Varnier, C., Zerhouni, N., Cherif, M., \& Fnaiech, N. (2017). Joint optimization of train assignment and predictive maintenance scheduling. In 7th international conference on railway operations modelling and analysis. Lille, France.

Huynh, K. T., Grall, A., \& Bérenguer, C. (2017). Assessment of diagnostic and prognostic condition indices for efficient and robust maintenance decision-making of systems subject to stress corrosion cracking. Reliability Engineering \& System Safety, 159, 237-254.

Kandukuri, S. T., Klausen, A., Karimi, H. R., \& Robbersmyr, K. G. (2016). A review of diagnostics and prognostics of low-speed machinery towards wind turbine farmlevel health management. Renewable and Sustainable Energy Reviews, 53, 697-708.

Khoury, E., Deloux, E., Grall, A., \& Bérenguer, C. (2013).
On the Use of Time-Limited Information for Maintenance Decision Support: A Predictive Approach under Maintenance Constraints. Mathematical Problems in Engineering, 2013, 1-11.

Ladj, A., Varnier, C., Tayeb, F. B. S., \& Zerhouni, N. (2017). Exact and heuristic algorithms for post prognostic decision in a single multifunctional machine. International Journal of Prognostics and Health Management, 8(2).

Langeron, Y., Grall, A., \& Barros, A. (2013). Actuator health prognosis for designing lqr control in feedback systems. Chemical engineering transactions, 33.

Langeron, Y., Grall, A., \& Barros, A. (2015, August). A modeling framework for deteriorating control system and predictive maintenance of actuators. Reliability Engineering \& System Safety, 140, 22-36.

Lebold, M., \& Thurston, M. (2001, January). Open standards for condition-based maintenance and prognostic systems. Maintenance and Reliability Conference (MARCON).

Lei, X., \& Sandborn, P. A. (2016). Phm-based wind turbine maintenance optimization using real options. International Journal of Prognostics and Health Management, 7(1), 1-14.

Lin, X., Basten, R. J. I., Kranenburg, A., \& van Houtum, G.-J. (2017). Condition based spare parts supply. Reliability Engineering \& System Safety, 168, 240-248.

Liu, Q., Dong, M., Lv, W., \& Ye, C. (2017, February). Manufacturing system maintenance based on dynamic programming model with prognostics information. Journal of Intelligent Manufacturing, 30(3), 1155-1173.

Moore, J. M. (1968). An n job, one machine sequencing algorithm for minimizing the number of late jobs. Management Science, 15(1), 102-109.

Mosallam, A., Medjaher, K., \& Zerhouni, N. (2016). Data-driven prognostic method based on bayesian approaches for direct remaining useful life prediction. Journal of Intelligent Manufacturing, 27(5), 1037 1048.

Nectoux, P., Gouriveau, R., Medjaher, K., Ramasso, E., Chebel-Morello, B., Zerhouni, N., \& Varnier, C. (2012). Pronostia: An experimental platform for bearings accelerated degradation tests. In Ieee international conference on prognostics and health management, phm'12. (pp. 1-8).

Nguyen, D. N., Dieulle, L., \& Grall, A. (2014, August). Feed- 
back control system with stochastically deteriorating actuator: Remaining useful life assessment. In Proceedings of the 19th world congress the international federation of automatic control. Cape Town, South Africa.

Nicolai, R. P., \& Dekker, R. (2008). Optimal maintenance of multi-component systems: A review. in: Complex system maintenance handbook. Springer, London: Springer Series in Reliability Engineering.

Pereira, E. B., Kawakami, R., Galvao, H., \& Yoneyama, T. (2010, July). Model predictive control using prognosis and health monitoring of actuators. International Symposium on Industrial Electronics, 237-243.

Rodrigues, L. R., Gomes, J. P. P., Ferri, F. A. S., Medeiros, I. P., Galvao, R. K. H., \& Nascimento Junior, C. L. (2015, December). Use of phm information and system architecture for optimized aircraft maintenance planning. IEEE Systems Journal, 9(4), 1197-1207.

Schwabacher, M. (2005). A survey of data-driven prognostics (Tech. Rep.). Aerospace Research Central.

Si, X.-S., Wang, W., Hu, C.-H., \& Zhou, D.-H. (2011). Remaining useful life estimation-a review on the statistical data driven approaches. European journal of operational research, 213(1), 1-14.

Sierra, G., Orchard, M., Goebel, K., \& Kulkarni, C. (2019). Battery health management for small-size rotary-wing electric unmanned aerial vehicles: An efficient approach for constrained computing platforms. Reliability Engineering \& System Safety, 182, 166-178.

Skima, H. (2016). Prognostics and distributed algorithms for post-prognostic decision making in mems based systems (Unpublished doctoral dissertation). FrancheComté University.

Skima, H., Varnier, C., Dedu, E., Medjaher, K., \& Bourgeois, J. (2017, February). Post-prognostics decision making in distributed mems-based systems. Journal of Intelligent Manufacturing.

Sun, B., Zeng, S., Kang, R., \& Pecht, M. (2010, January). Benefits analysis of prognostics in systems. 2010 Prognostics and System Health Management Conference, $1-10$.

Tamilselvan, P., \& Wang, P. (2013). Failure diagnosis using deep belief learning based health state classification. Reliability Engineering \& System Safety, 115,
124-135.

Uckun, S., Goebel, K., \& Lucas, P. J. (2008, October). Standardizing research methods for prognostics. In International Conference on Prognostics and Health Management, 2008. PHM 2008., 1-10.

Vachtsevanos, G., \& Wang, P. (2001). Fault prognosis using dynamic wavelet neural networks. In In autotestcon proceedings, 2001. ieee systems readiness technology conference (pp. 857-870). IEEE.

Van Horenbeek, A., \& Pintelon, L. (2013). A dynamic predictive maintenance policy for complex multi-component systems. Reliability Engineering \& System Safety, 45(50), 39-50.

Vieira, J. P., Kawakami, R., Galvao, H., \& Yoneyama, T. (2015, December). Predictive control for systems with loss of actuator effectiveness resulting from degradation effects. Journal of Control Automation and Electrical Systems, 26(6), 589-598.

Vileiniskis, M., \& Remenyte-Prescott, R. (2017). Quantitative risk prognostics framework based on petri net and bow-tie models. Reliability Engineering \& System Safety, 165, 62-73.

Wang, Z., Hu, C., Wang, W., Kong, X., \& Zhang, W. (2015). A prognostics-based spare part ordering and system replacement policy for a deteriorating system subjected to a random lead time. International Journal of Production Research, 53(15), 4511-4527.

Welz, Z., Coble, J., Upadhyaya, B., \& Hines, W. (2017, August). Maintenance-based prognostics of nuclear plant equipment for long-term operation. Nuclear Engineering and Technology, 49(5), 914-919.

Wu, G., Vachtsevanos, F., Lewis, M., Roemer, A., \& Hess, B. (2006). Intelligent fault diagnosis and prognosis for engineering systems. Wiley, Hoboken, NJ.

Yang, Z. M., Djurdjanovic, D., \& Ni, J. (2008, February). Maintenance scheduling in manufacturing systems based on predicted machine degradation. Journal of Intelligent Manufacturing, 19(1), 87-98.

Zhang, B., Tang, L., Decastro, J., Roemer, M., \& Goebel, K. (2014, July). Autonomous vehicle battery state-ofcharge prognostics enhanced mission planning. International Journal of Prognostics and Health Management, 5(8). 